\title{
Nasopharyngeal Carcinoma Metastatic to the Mandible
}

\author{
Po Hsien Huang ${ }^{1}$, Yih Chuen Shyng ${ }^{2}$, P Sloan ${ }^{3}$, Keng-Liang Ou ${ }^{4}$, Y. J. Hsia ${ }^{5}$ and H. Devlin ${ }^{*}, 6$ \\ ${ }^{I}$ Chief Resident of Kaoshiuang Military General Hospital, Taiwan \\ ${ }^{2}$ Head of Medical Research and Animal Study Unit, Kaohsiung Military General Hospital, 2 Chengchung $I^{\text {st }}$ Rd., Kaoh- \\ siung Taiwan \\ ${ }^{3}$ School of Dentistry, University of Newcastle, UK \\ ${ }^{4}$ Graduate Institute of Oral Sciences, College of Oral Medicine, Taipei Medical University, Taipei 110, Taiwan \\ ${ }^{5}$ Head of Department of Oral and Maxillofacial Surgery, Tri-Service General Hospital, Taiwan \\ ${ }^{6}$ School of Dentistry, University of Manchester, Higher Cambridge St., Manchester M15 6FH, UK
}

\begin{abstract}
Nasopharyngeal carcinoma (NPC) is one of the most common malignancies in the head and neck region, especially among those of Chinese origin. NPC has multifactorial aetiologies including genetic susceptibility, consumption of food with high salt content, and the Epstein-Barr virus. The primary tumour usually arises from the lateral walls of the nasopharynx and is characterized by a rich sub-mucosal lymphatic structure, often leading to cervical lymph node metastasis. Distant metastasis has been recognized to be a major cause of treatment failure in patients with nasopharyngeal carcinoma. Bone, liver and lung are the most frequent sites of NPC metastases.
\end{abstract}

Keywords: Mandible, Nasopharyngeal carcinoma.

\section{INTRODUCTION}

We report an unusual case of NPC that was metastatic to the mandible. Others have reported similar unusual presentations with symptoms that have developed in the maxillofacial region. Recently, Khan et al. [1] reported a patient that presented with facial pain of three months duration and limited mouth opening. MRI and a CT scan revealed a large NPC in the nasopharyngeal space. NPC metastasis to the parotid gland is extremely rare, but may present as a parotid swelling [2].

It is generally a rare malignancy in developed countries, but has a higher incidence in Southern China and Southeast Asia. Its incidence in different countries is related to EpsteinBarr infection rates, with additional smaller environmental effects such as smoking and genetic susceptibility playing a role [3]. Failure of treatment for NPC often occurs because of distant metastases, which is therefore an important factor determining survival. Metastasis, in the absence of local recurrence, implies occult dissemination at the initial presentation [4].

\section{CASE REPORT}

A 79 year-old male was referred to our hospital with pus discharging for one month from an unhealed intraoral wound in the mandibular symphysis area. The panoramic radiograph showed that there was a 4 x $3 \mathrm{~cm}$ size radiolucent lesion over the mandibular symphysis with an irregular border (Fig. 1).

*Address correspondence to this author at the School of Dentistry, University of Manchester, Higher Cambridge St., Manchester M15 6FH, UK; Tel: +44(0)-161-275-6849; Fax: +44(0)-161-275-6710;

E-mail: Hugh.Devlin@Manchester.ac.uk

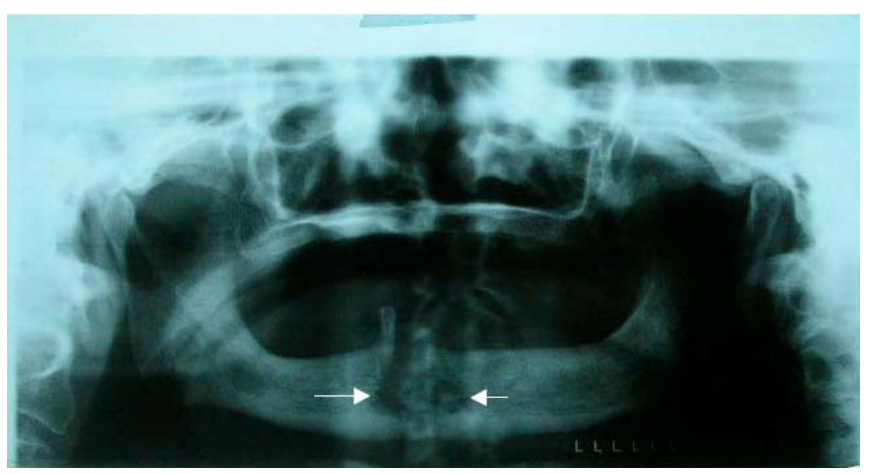

Fig. (1). A large radiolucent lesion (between arrows) and a retained tooth in the anterior area of mandible at the patient's first visit to our hospital.

There was no palpable lymphadenopathy over the bilateral neck area during the physical examination. The patient had a history of NPC one year previously, and had received a complete radiation therapy course (total dose of $6500 \mathrm{~Gy}$ ), without chemotherapy. A tentative diagnosis of osteomyelitis of the mandible was made. In order to eliminate the possibility of NPC metastasis, a chest radiograph, conventional bone scanning and liver scanning were arranged. The results were negative. CT scanning did not reveal any cervical lymph node involvement in the neck area. Under general anaesthesia, tooth extraction, sequestrectomy and marginal bone resection were performed with a $2 \mathrm{~mm}$ safe zone (Fig. 2). The tissue specimen was whitish in colour and soft with a granular texture. The wound was marsupialized and an iodoform gauze dressing was placed for 2 weeks. The histopathology report showed an undifferentiated carcinoma with frequent mitoses. In-situ hybridization revealed that the 


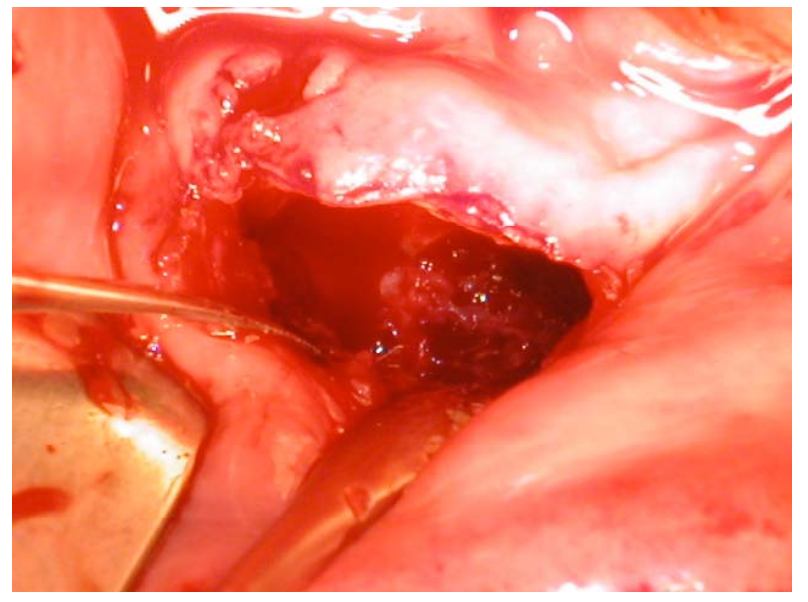

Fig. (2). Enucleation and marginal bone resection were performed.

specimen contained Epstein-Barr virus RNA. The ENT department performed a nasopharyngeal examination for the possibility of recurrent NPC and the result was negative. The case was classified as Ho's stage II, T1N1M1.

A CT scan was taken one month after the operation. The mandible had healed in the upper border region, but no heal- ing was evident over the lower margin after surgery (Fig. 3). The patient was referred for radiotherapy. After the supplemental dose of $50 \mathrm{~Gy}$, further lesion expansion was stopped and early bone formation in the inferior lingual aspect of the lesion was noted in the following CT scan (Fig. 4). The remaining apparent discontinuity of the bone in the grafted area most probably represents incomplete healing. 6 months after the major surgery, the patient complained of chest pain, and received a further chest CT scan. A lung metastasis was diagnosed. The patient was then referred to receive salvage chemotherapy with 5-flurouracial and cisplatin.

\section{DISCUSSION}

NPC is considered as surgically unresectable because of its anatomical localization. It is primarily treated by radiotherapy. Distant metastasis has been found to be the major cause of mortality with NPC. $5 \%$ of patients already have distant metastases at presentation and up to $30 \%$ are found to have distant metastases after primary definitive radiotherapy. $60-80 \%$ of patients have metastatic lymphadenopathy.

In our patient the radiological examination with whole body bone scan, chest radiography and liver scan failed to detect the distant metastasis early. In treatment choices, NPC is a highly radiosensitive tumour and radical external radio-
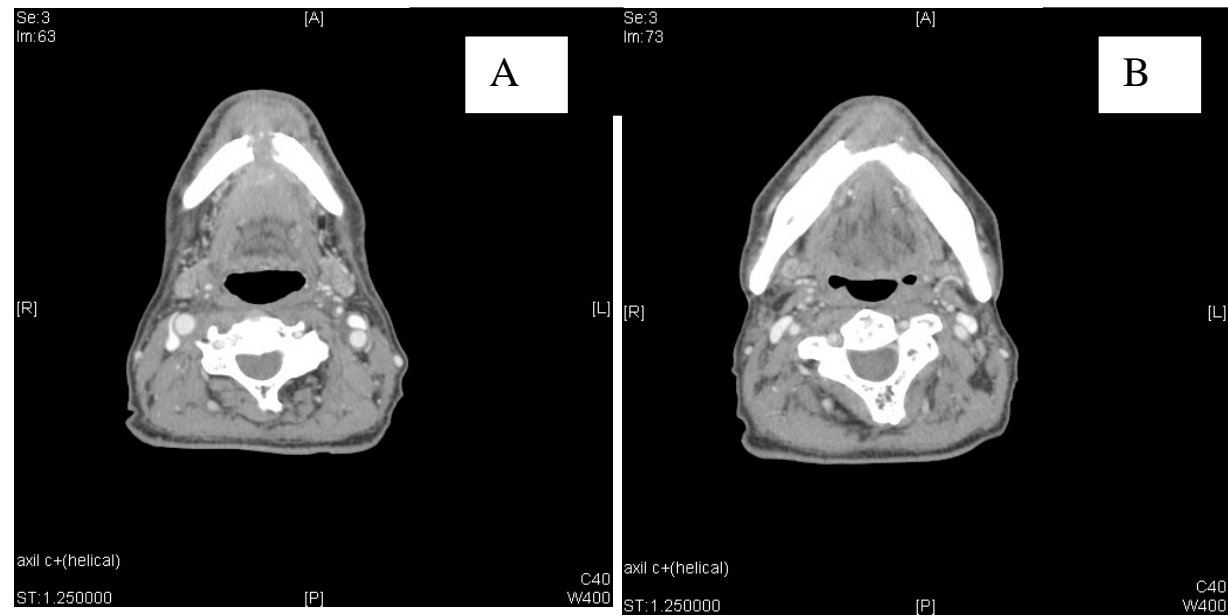

Fig. (3). CT scan taken one month after operation showing a defect in the anterior mandible (A) with a bridge of bone on the superior aspect (B).
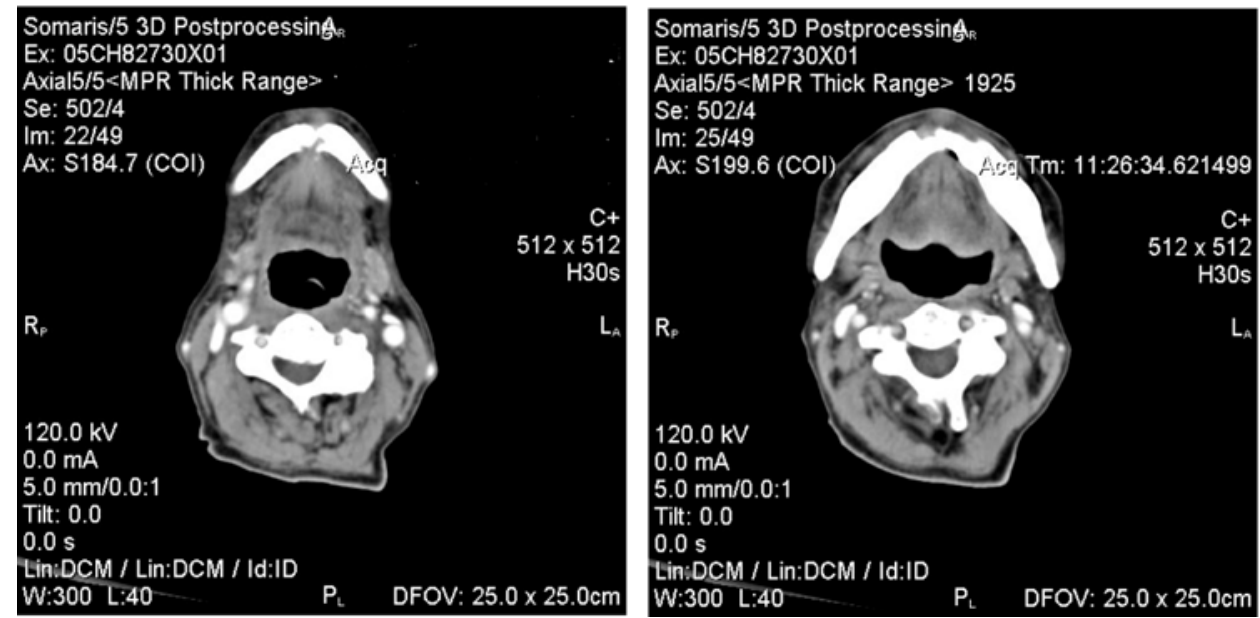

Fig. (4). CT scan 5 months post-operation after salvage radiotherapy showing increased bone formation and a decrease in the residual lesion. 
therapy results in an overall 5-year survival rate of over $80 \%$ for Ho's Stage I and over 70\% for Ho's Stage II disease [510] Chemotherapy is effective in locoregionally recurrent or metastatic NPC disease. Distant metastasis is frequent in bone, but facial bone involvement has been rarely reported.

Could the surgical intervention of the oral lesion have contributed to the dissemination of the cancer to the lungs? The surgical intervention to the jaw was not likely to have caused the lung metastasis. A throat pack was used and care was taken to avoid aspiration of tumour cells. It is most likely that the lung metastasis was a late clinical manifestation of blood borne dissemination occurring earlier in the course of the disease. The muco-ciliary barrier is effective and malignant cells need to establish a blood supply to survive. The patient eventually developed a solitary pulmonary metastasis. Options for management in this situation include local surgical removal and salvage chemotherapy. The evidence base for surgical removal of metastasis is weak, and is most useful for cancers with a prolonged clinical course and without wide dissemination. It is widely accepted that nasopharyngeal carcinoma disseminates early and the evidence available indicates that salvage is best attempted using platinum based chemotherapy.

The mandible may also be affected by osteoradionecrosis, which is a rare complication of radiotherapy treatment of head and neck tumours. The features of soft tissue thickening and cortical bone destruction may lead to an erroneous diagnosis of tumour recurrence [11]. The risk of osteoradionecrosis is greatest with high doses of radiation in patients with poor oral hygiene. The buccal cortex in the molar and premolar regions are most commonly affected, while the symphysis and angle of the mandible are protected because of the blood supply derived from their associated muscle insertions. The trismus, dryness of the oral mucosa and fibrosis of sulcular tissues that may follow radiotherapy, can complicate examination of the patient's mouth and any restorative treatment $[12,13]$.

NPC is primarily treated by radiotherapy, and postirradiation osteosarcoma (PIOS) arises as a late complication in about $0.04 \%$ of people treated. PIOS in NPC is a highly malignant disease, which occurs after a prolonged latency period following radiotherapy. Wei-wei, et al. [14] found that this latency period was about 13 years on average after irradiation in their group of patients. The prognosis of radiation-induced sarcoma is worse than that of the primary disease [15]. In a group of 12 patients with PIOS following irradiation for NPC, survival ranged from 7 to 41 months, with a mean of 18 months [16].

The patient's initial presentation of NPC that was metastatic to the mandible is unusual, with pus discharging for a month from an unhealed intraoral wound. In a retrospective analysis of 4768 patients with primary NPC, Lee, et al. [17] found that the most common presenting complaint was a painless enlargement of the upper cervical lymph nodes, nasal symptoms (such as discharge, bleeding or obstruction), otological problems, headache or cranial nerve dysfunction. The importance of early detection is emphasized.

\section{REFERENCES}

[1] Khan J, Quek SY, Markman S. Nasopharyngeal carcinoma masquerading as TMJ orofacial pain. Quintessence Int 2010; 41: 387-9.

[2] Dong XR, Zhang T, Fan L, Zhang S, Wu G. Parotid gland metastasis of nasopharyngeal carcinoma: case report and review of the literature. J Int Med Res 2009; 37: 1994-9

[3] Mousavi SM, Sundquist J, Hemminki K. Nasopharyngeal and hypopharyngeal carcinoma risk among immigrants in Sweden. Int J Cancer 2010; 19(4): 275-9.

[4] Teo PML, Kwan WH, Lee WY, Leung SF, Johnson PJ. Prognosticators determining survival subsequent to distant metastasis from nasopharyngeal carcinoma. Cancer 1996; 77: 2423-31.

[5] Marks JE, Bedwinek JM, Lee F, Purdy JA, Perez CA. Dose response analysis for nasopharyngeal carcinoma. Cancer 1982; 50:104250 .

[6] Qin D, Hu Y, Yan J, et al. Analysis of 1379 patients with nasopharyngeal carcinoma treated by radiation. Cancer 1988; 61:111724.

[7] Wang CC, Meyer JE. Radiotherapeutic management of carcinoma of the nasopharynx: analysis of 170 patients. Cancer 1971; 28: 56670 .

[8] Ho JHC. Stage classification of nasopharyngeal carcinoma: a review. In: de Tha G, Ito Y, Eds. Nasopharyngeal carcinoma aetiology and control. Lyon, France: IARC Scientific Publications 1978; 99.

[9] Teo PML, Tsao SY, Ho JHC, Yu P. A proposed modification of the Ho-stage classification for nasopharyngeal carcinoma. Radiother Oncol 1991; 21: 11-23.

[10] Sham JST, Choy D. Prognostic factors of nasopharyngeal carcinoma: a review of 759 patients. Br J Radiol 1989; 63: 51-8.

[11] Hermans R, Fossion E, Ioannides C, Van den Bogaert W, Ghekiere J, Baert AL. CT findings in osteoradionecrosis of the mandible. Skeletal Radiol 1996; 25: 31-6.

[12] Devlin H, Barker GR. Prosthetic and surgical care of radiotherapy patients by the general dental practitioner. Dental Update 1988; 16: 166-9.

[13] Chu FC, Wong WK, Wong YK, Chow BK, Cheng JC. Implant treatment of macroglossia and edentulous mandible following radiotherapy for nasopharyngeal cancer: a case report. Quintessence Int 2003; 34: 670-3.

[14] Wei-wei L, Qiu-liang W, Guo-hao W, Zhi-hua C, Zong-yuan Z Clinicopathologic features, treatment, and prognosis of postirradiation osteosarcoma in patients with nasopharyngeal cancer. Laryngoscope 2005; 115: 1574-9.

[15] Chabchoub I, Gharbi O, Remadi S, et al. Postirradiation osteosarcoma of the maxilla: a case report and current review of literature. $\mathbf{J}$ Oncol 2009; 2009: 876138.

[16] Liu WW, Chen ZH, Wu QL, Wu GH, Zeng ZY. Clinicopathological features and prognosis of postirradiation osteosarcoma in nasopharyngeal carcinoma. Zhonghua Er Bi Yan Hou Tou Jing Wai Ke Za Zhi 2005; 40: 814-8.

[17] Lee AW, Foo W, Law SC, et al. Nasopharyngeal carcinoma: presenting symptoms and duration before diagnosis. Hong Kong Med J 1997; 3: 355-61. 